\title{
Clinicians' Core Needs in a Pandemic: Qualitative Findings From the Chat Box in a Statewide COVID-19 ECHO Program
}

\author{
Anna L. Steeves-Reece, MPH, $M A^{1,2}$ \\ Nancy C. Elder, MD, MSPH ${ }^{1,3}$ \\ Katherine D. Broadwell ${ }^{1}$ \\ Ronald D. Stock, $M D, M A^{1}$ \\ 'Oregon Rural Practice-based Research \\ Network, Oregon Health \& Science University, \\ Portland, Oregon \\ ${ }^{2}$ Oregon Health \& Science University-Portland \\ State University School of Public Health, Port- \\ land, Oregon \\ ${ }^{3}$ Oregon Health \& Science University Depart- \\ ment of Family Medicine, Portland, Oregon
}

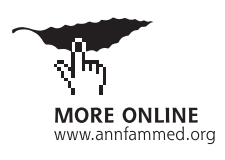

Conflicts of interest: authors report none.

\section{CORRESPONDING AUTHOR}

Anna L. Steeves-Reece 3181 SW Sam Jackson Park Rd

Portland, OR 97239

steevesr@ohsu.edu

\begin{abstract}
PURPOSE Research on primary care's role in a pandemic response has not adequately considered the day-to-day needs of clinicians in the midst of a crisis. We created an Oregon COVID-19 ECHO (Extension for Community Healthcare Outcomes) program, a telementoring education model for clinicians. The program was adapted for a large audience and encouraged interactivity among the hundreds of participants via the chat box. We assessed how chat box communications within the statewide program identified and ameliorated some of clinicians' needs during the pandemic.
\end{abstract}

METHODS We conducted a qualitative analysis of chat box transcripts from 11 sessions. We coded transcripts using the editing method, whereby analysts generate categories predominantly from the data, but also from prior knowledge. We then explored the context of clinicians' needs in a pandemic, as conceptualized in Maslow's hierarchy of needs adapted for physicians: physiologic, safety, love and belonging, esteem, and self-actualization.

RESULTS The mean number of chat box participants was 492 per session (range, 385 to 763). Participants asked 1,462 questions and made 819 comments throughout the program. We identified 3 key themes: seeking answers and trustworthy information, seeking practical resources, and seeking and providing affirmation and peer support. These themes mapped onto the Maslow's needs framework. We found that participants were able to create a virtual community in the chat box that supported many of their needs.

CONCLUSIONS Using a novel data source, we found sharing the experience of practicing in a rapidly changing environment via comments and questions in an ECHO program both defined and supported participants' needs.

Ann Fam Med 2022;20:51-56. https://doi.org/10.1370/afm.2762.

\section{INTRODUCTION}

$\Lambda^{\mathrm{s}}$ COVID-19 moved across the United States in early 2020, primary care clinicians faced dramatic changes to their practices, often with little assistance and inconsistent guidance. ${ }^{1}$ Without a unified national response, states were forced to create their own policies regarding how health care would be provided and paid for. ${ }^{2}$ Although the initial focus in the country was on hospitals, primary care practices also needed rapid support and accurate information.

The role of primary care has not typically been considered in discussions of pandemic preparations. In 2005, Osterholm ${ }^{3}$ posited planning for the next pandemic would require ramped-up vaccine production, antiviral drug prioritization, and a detailed operational plan for public health, but did not mention primary care at all. More recent articles describe how primary care may contribute more to pandemic care; they suggest partnering with public health for prevention, diagnosis, treatment, and leveraging of the Centers for Disease Control and Prevention pandemic framework to ensure primary care can complete its main work along with pandemic tasks. ${ }^{4,5}$ These discussions, however, focus on primary care as a discipline rather than on individual clinicians navigating the pandemic on a day-to-day basis.

Identifying and meeting clinicians' needs during a pandemic, or any public health crisis, is key for primary care as a discipline to reach its full potential. To help define these needs, researchers have adapted Maslow's hierarchy of needs to physicians. ${ }^{6-8}$ Maslow's 1943 framework posits humans have 5 levels of need: physiologic, safety, love and belonging, esteem, and self-actualization. ${ }^{9}$ When applied to physicians, physiologic needs include food, sleep, and health, while safety includes physical as well as financial security. Respect is a component of either love and 
belonging ${ }^{8}$ or esteem, ${ }^{6}$ along with appreciation and connection to others. Self-actualization relates to caring for patients and contributing to the fullest of one's abilities. Hale et $\mathrm{al}^{6}$ posit these needs are not hierarchical, but support each other and create an integrated framework.

Our team explored the following question: What can we learn about clinicians' needs during the COVID-19 pandemic by analyzing chat box comments from online learning sessions? The sessions were part of a telementoring education model called Project ECHO (Extension for Community Healthcare Outcomes). In March 2020, to rapidly disseminate emerging public health and clinical best practices, we created an 11-week COVID-19 ECHO for frontline clinicians. While modifying the program for an anticipated large audience, it was important to sustain a format that was more interactive and responsive than a traditional webinar; we did this by maximizing the use of the chat box.

Over the 11-week COVID-19 ECHO program, we observed that participants' use of the chat box went well beyond asking clinical questions, including the emergence of a support network. This article presents a qualitative analysis of chat box data, examining clinicians' questions and comments. We used adaptations of Maslow's framework to interpret expressions of clinicians' needs during this health care crisis.

\section{METHODS}

\section{The COVID-19 ECHO Program}

Project ECHO is a telementoring education model that expands primary care clinicians' ability to manage complex health conditions. An interdisciplinary faculty, an "all teach, all learn" approach, and case-based learning characterize ECHO programs. ${ }^{10,11}$ The Oregon ECHO Network (OEN) offers a variety of programs and resides within the Oregon Rural Practice-based Research Network. ${ }^{12}$ At the start of the pandemic, we recognized ECHO could effectively disseminate pertinent information to clinicians across the state. As we previously described ${ }_{1}^{13}$ we relied on OEN's established systems and relationships to identify credible faculty experts and widely promote the program.

The first of the 11 weekly ECHO sessions was held March 19, 2020. Each session lasted 75 minutes and began with an update from regional public health experts on case numbers, hospitalizations, deaths, and the governor's executive orders. Next, an expert presenter (eg, an infectious disease or immunology specialist), a community presenter from a practice, or both shared updates and best practices. Moderated questions followed each presentation (see Supplemental Table 1 for ECHO topics and speakers).

We used the chat box in multiple ways. First, participants used it to submit their questions for speakers. Primary care physician staff monitored the chat box and synthesized key questions. As there were more questions asked than could be answered during each session, immediately afterward, the chat box (15 to 20 pages of text) was reviewed and summarized by 2 OEN staff members and 2 physicians who had monitored the chat box during the session. Our team posted this chat box summary to the registration platform. The chat box content also informed which topics to include in future sessions. In addition, we partnered with Oregon Health \& Science University's COVID-19 Inquiry Group to have the $\mathrm{ECHO}$ questions added to the list of questions for which they researched answers for patients and clinicians. ${ }^{14}$ We posted these answers on our registration platform, available to registered $\mathrm{ECHO}$ participants.

\section{Participants}

Health care professionals who registered for the ECHO program provided demographics and details about their professional roles. Registration was not a prerequisite to participate in the sessions, but incentives included calendar reminders and qualifying to receive free continuing medical education credits.

\section{Data Source and Analysis}

Our primary data sources were the chat box transcripts from the $11 \mathrm{ECHO}$ sessions. Three qualitative analysts with respective backgrounds in family medicine, public health, and medical anthropology (N.C.E., A.L.S-R., and K.D.B.) were involved in monitoring the chat box during sessions and thus familiar with its real-time use. We used ATLAS.ti 8 Windows (Scientific Software Development $\mathrm{GmbH}$ ) to code chat box transcripts using the editing method, whereby the coding categories are created from the data themselves, but are informed by preexisting knowledge and experiences. ${ }^{15}$ Two analysts (N.C.E. and K.D.B.) coded the same 2 sessions and then met to align their coding assignments, coming to agreements on codes and categories. One analyst then coded all transcripts, while the other analyst read each transcript for initial themes. All 3 analysts then met several times, discussing prevalent themes and reviewing coding patterns. They then discussed these themes in light of Maslow's hierarchy of needs. Finally, 2 analysts (N.C.E. and K.D.B.) returned to the coding categories to affirm these discussions in the context of clinicians' needs during the pandemic, adapting them to the literature on Maslow's framework for physicians. The institutional review board at Oregon Health \& Science University determined all OEN programs and their evaluations to be "not human research."

\section{RESULTS}

\section{Participation}

A total of 1,275 health professionals registered at some time during the 11-week COVID-19 ECHO program (Table 1). Registrants came from all of Oregon's 36 counties. The majority of registrants reported providing direct patient care and working in a primary care setting. Physicians and nurse practitioners accounted for nearly one-half of registrants. Attendance at each chat box session ranged from 385 to 763 (mean, 492). Attendance was highest for the first 4 sessions. 


\section{Table 1. Characteristics of Health Care Professionals Registering for the COVID-19 ECHO $(\mathrm{N}=1,275)$}

\begin{tabular}{|c|c|}
\hline Characteristic & Registrants, No. (\%) \\
\hline \multicolumn{2}{|l|}{ Age group, ${ }^{a}$} \\
\hline $20-39$ & $373(29.3)$ \\
\hline $40-59$ & $664(52.1)$ \\
\hline$\geq 60$ & $238(18.7)$ \\
\hline Total & $1,275(100.0)$ \\
\hline \multicolumn{2}{|l|}{ Self-reported gender } \\
\hline Female/woman & $902(70.8)$ \\
\hline Male/man & $310(24.3)$ \\
\hline Prefer not to respond & $53(4.2)$ \\
\hline $\begin{array}{l}\text { Gender queer/gender nonconforming } \\
\text { or transgender }\end{array}$ & $10(0.8)$ \\
\hline Total & $1,275(100.0)$ \\
\hline \multicolumn{2}{|l|}{ Self-reported race/ethnicityc } \\
\hline American Indian or Alaska Native & $18(1.4)$ \\
\hline Asian or Asian American & $92(7.2)$ \\
\hline Black or African American & $13(1.0)$ \\
\hline Hispanic or Latinx & $54(4.2)$ \\
\hline Other & $31(2.4)$ \\
\hline Prefer not to respond & $118(9.3)$ \\
\hline White & $965(75.7)$ \\
\hline \multicolumn{2}{|l|}{ Population served } \\
\hline Rural or frontier & $337(26.4)$ \\
\hline Provide direct patient care & $984(77.2)$ \\
\hline Primary care setting & $788(61.8)$ \\
\hline Workplace accepts Medicaid & $834(65.4)$ \\
\hline \multicolumn{2}{|l|}{ ECHO participation history } \\
\hline First-time ECHO participant & $1,061(83.2)$ \\
\hline \multicolumn{2}{|l|}{ Marketing source } \\
\hline E-mail & $771(60.5)$ \\
\hline Word of mouth & $233(18.3)$ \\
\hline Other/multiple sources & $270(21.2)$ \\
\hline Total & $1,274(100.0)$ \\
\hline \multicolumn{2}{|l|}{ Profession } \\
\hline Physician & $476(37.3)$ \\
\hline Nurse practitioner & $154(12.1)$ \\
\hline Administrative staff & $98(7.7)$ \\
\hline Physician assistant & $81(6.4)$ \\
\hline Behavioral health professional & $74(5.8)$ \\
\hline Pharmacist & $34(2.7)$ \\
\hline Nursing & $31(2.4)$ \\
\hline Nursing facility staff & $30(2.3)$ \\
\hline Health coordinator/educator & $25(2.0)$ \\
\hline Other & $272(21.3)$ \\
\hline Total & $1,275(100.0)$ \\
\hline
\end{tabular}

$\mathrm{ECHO}=$ Extension for Community Healthcare Outcomes.

a Roughly calculated from birth year.

b Categories combined to protect personal identifiers.

' Race/ethnicity categories do not add up to $100 \%$. We present the number of registrants who selected each category, and multiple categories were permitted; roughly, $8.6 \%$ of registrants selected more than 1 category.
On the basis of an analysis of unique screen names within a session, $15 \%$ to $20 \%$ of participants in each session contributed to the chat box conversation.

\section{Summary of Chat Box Use \\ Activity}

The chat box was mainly used to prioritize and answer participants' questions for presenters. Beginning in the first session, however, participants began making comments as well. They publicly responded to each other with references to articles they had read or examples from their personal experiences. They validated questions asked with, "Yes, I want to know that, too," and they referred each other to regional resources for everything from personal protective equipment (PPE) to small business loans. They also expressed their personal and professional fears and concerns.

\section{Questions}

Participants asked 1,462 questions across the sessions, with a range of 74 to 216 questions per session and a mean of 132. The most frequent questions pertained to acute testing, transmission, disparities in COVID-19, and PPE. Questions about COVID-19 pathophysiology, disease symptoms, and treatment were also common, as were questions about how to provide regular primary care during the pandemic. Questions varied by topics covered in the session. For example, there were more questions about treating substance use disorders during the session when addiction medicine faculty gave a presentation.

\section{Comments}

Participants made 819 comments in total, with a range of 31 to 131 comments per session and a mean of 75 . We found 4 main comment types: those sharing references, guidelines, and other resources; those answering or commenting to other participants; those sharing personal experiences; and those we could not classify into any of the previous categories ("miscellaneous" or "unable to tell"). Comments of the last type were most frequently about the federal and state response, political or social comments, or other clinical health issues.

\section{Chat Box Themes}

We identified 3 themes across the questions and comments in the chat box transcripts: (1) seeking answers and trustworthy information; (2) seeking practical resources; and (3) seeking and providing affirmation and peer support. We detail each of these themes in the following sections.

\section{Seeking Answers and Trustworthy Information}

Participants used the chat box most commonly to ask questions of the presenters or to raise clinical questions from their experiences or their reading of both medical and social media. Participants asked for scientific details and clinical updates about COVID-19 transmission, testing, and symptoms. They also sought to understand and highlight the 
pandemic's impact on those affected by structural vulnerability, including racial and ethnic disparities in COVID-19 cases, hospitalizations, and mortality. There were many questions about PPE effectiveness and use, for example, "In the primary care setting, how do you determine when to don PPE (that is severely limited) for complaints of cough and shortness of breath?"

Contact tracing questions began appearing more commonly in the seventh week. Participants also shared references, guidelines, and articles with each other. They expressed concerns about keeping up with rapidly changing information, often feeling unable to care for patients properly during the pandemic. For example, one participant commented, "Trying to keep up with changing information-it's a huge sense of responsibility to keep up."

\section{Seeking Practical Resources}

From the first session onward, participants shared practical resources with each other, and the OEN team recruited both regional experts and community presenters who could further share recommendations and personal stories. Presentations covered many topics, such as drive-through patient visits and testing, telehealth, and end-of-life care.

A common thread expressed by participants was that the "response is very fragmented, it feels like every clinic and system is on their own." They wondered where to get and how to care for $\mathrm{PPE}_{i}$ how to use telehealth technology, workflows, and billing; how to care for non-COVID-19 clinical issues; and how to get supplies for COVID-19 testing. In addition to the ECHO speakers, participants offered their own recommendations in the chat box: "I use [company name] for cloud-based faxes and added the telemedicine service, which has been easy. Even my mom without instructions was able to do it."

\section{Seeking and Providing Affirmation and Peer Support}

Participants consistently used the chat box to communicate with each other. As they shared their thoughts about emerging articles and personal experiences of working through clinical issues, they had an opportunity to receive affirmation from their peers. They confirmed that they, too, had many of the same questions and confusions. Questions and comments would begin with, "I also read the New York Times piece and I'm concerned..." and "Same question—will we learn..."

The chat box was also a venue to share, or raise concerns, about the state and federal responses to COVID-19. It was a place for participants to express frustration, anger, and fear about living and working in a pandemic. Beyond concerns about catching COVID-19 themselves, financial fears were common. For example, "I am a physician and I was terminated from my job as a result of this," and, "I'm in private practice-only having a few video visits/week. How do I pay staff?" In addition, the family stresses and the loss of human connection affected participants, as one expressed: "With the switch to telemedicine, I really miss the in-person connections with patients."

\section{Chat Box Themes Applied to Maslow's Adapted Hierarchy of Needs}

We further analyzed our qualitative findings by mapping the 3 main themes onto Maslow's adapted hierarchy of needs for physicians ${ }^{7}$ as shown in Figure 1. The levels physiologic and safety were clearest in the theme of seeking practical resources, but were also touched on in affirmation and peer support. Participants asked for and shared with each other their most basic needs for safety and security. They expressed concerns about their emotional and physical health, such as fatigue from living with ongoing uncertainty and change. They demonstrated a desire for personal safety by seeking PPE to protect themselves from infection and illness. Participants shared about salary and job losses, and figuring out how to bill appropriately for telehealth reimbursement was a key way participants sought financial security.

Maslow's next 2 levels, love and belonging, and esteem, include relationships with family and friends, respect of colleagues and peers, being appreciated, and having connections. We saw these needs addressed in the theme of affirmation and peer support. At a time when participants were concerned about their families and their loss of connections, their colleagues provided connection and validation through the chat box. By providing resources and references in the chat box, they had an opportunity to earn the respect of their peers. Discussing their fears and concerns connected them with others experiencing the same emotions. The ability to air grievances with everything from the government to the media provided support and an ability to take back some control in a time when uncertainty was overwhelming.

Maslow's highest level is self-actualization, which, when adapted for physicians, includes the capacity to heal, connect, and reach the full development of one's abilities. Enhancing participants' ability to practice medicine during the pandemic is what led to the creation of the COVID-19 ECHO program and was likely an initial motivator for most of the participants - our theme of seeking answers and trustworthy information. The sheer number and diversity of questions asked demonstrated how important this objective was to participants. We also saw many participants taking on an advocacy role, another demonstration of self-actualization. Participants advocated within the chat box for their marginalized or underserved patients (eg, houseless people, migrant workers, those affected by racism) and for those with special health needs (eg, individuals with substance use disorders, the elderly). They asked medical and policy questions and advocated for answers and updates regarding these patients and issues.

\section{DISCUSSION}

Through analyzing chat box transcripts from a COVID-19 ECHO program, we identified core themes regarding clinicians' needs in the early stages of the pandemic. We were also able to map those themes onto an adapted framework of needs for physicians. Finally, our results describe the ways 
Figure 1. Illustrative quotes embedded within the chat box themes and Maslow's hierarchy of needs adapted for physicians.

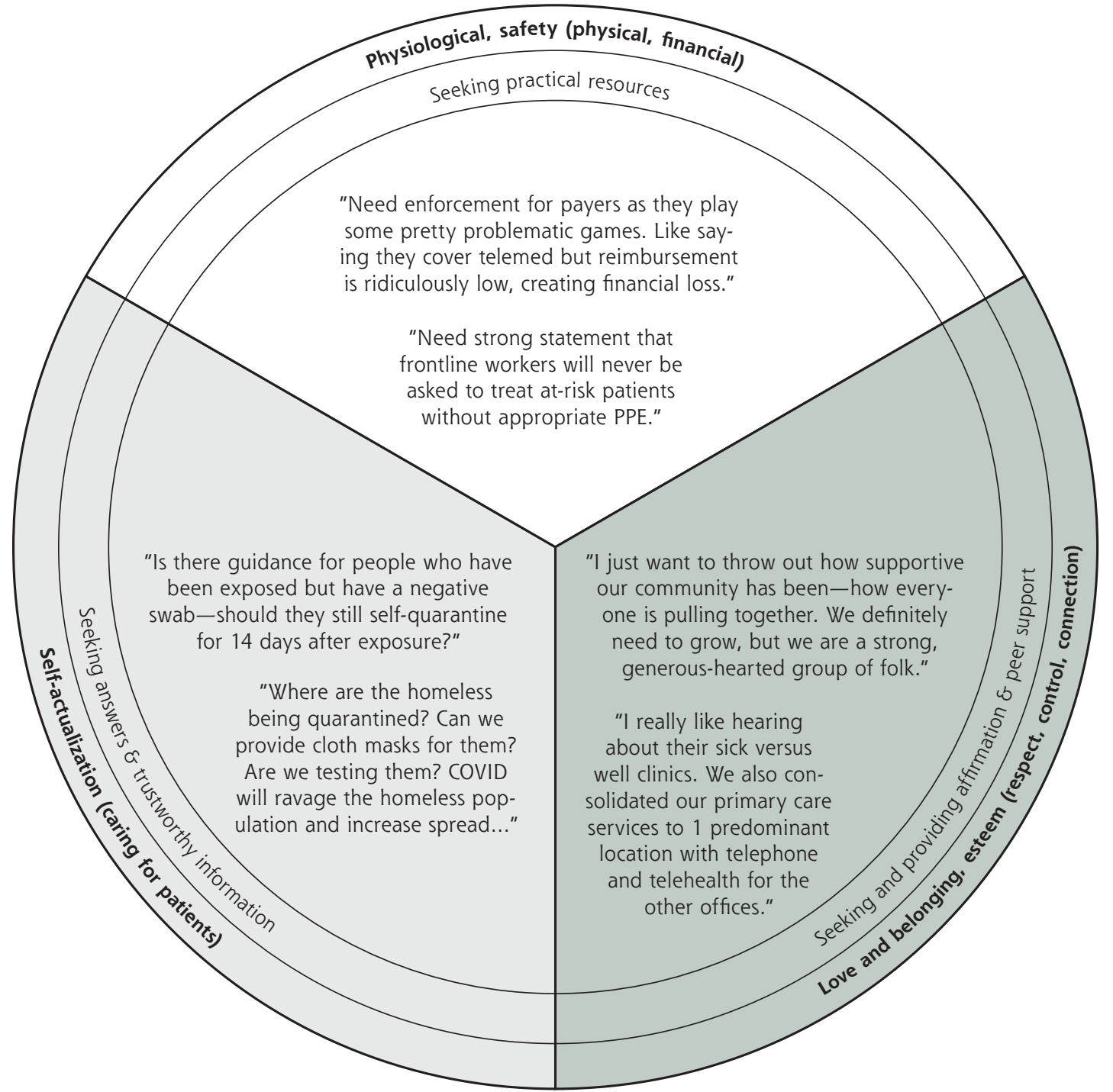

PPE = personal protective equipment.

Note: The outer ring shows Maslow's needs for physicians. The inner ring shows the chat box themes that best mapped onto the respective Maslow's needs categories. The core shows quotes related to both the chat box themes and Maslow's needs.

in which the ECHO program was able to address some of clinicians' needs. Using this novel data source, we confirmed that primary care clinicians, the first point of contact in the health care system for most Americans ${ }^{16}{ }^{16}$ often felt they were on their own to find everything from the answers to clinical questions to PPE sources. ${ }^{1}$

Like other organizations, ${ }^{17}$ we quickly realized the $\mathrm{ECHO}$ model had great potential to support clinicians during the pandemic..$^{13}$ Although summarizing the chat box and sharing a few frequently asked questions with presenters was far from the open conversation typical of the ECHO model ${ }_{1}^{18}$ we discovered participants still created a learning community. Peerto-peer learning and collaborative problem solving took place in all 3 thematic areas. In our analysis, we found participating clinicians were not just describing, but also meeting some of their needs as detailed in an adapted Maslow's framework. A key task for the future is to find ways to maintain this support for clinicians through Project ECHO and similar programs.

The COVID-19 pandemic is unprecedented in the lives of current clinicians, and little of the suggested infrastructure for such an event was put into place in the past 20 years. ${ }^{3}$ As public health and primary care experts plan for the next pandemic (or other crises), our findings add to those recommending preparations for and redesign of primary care in this country. ${ }^{4,5,19}$ Clinicians' basic needs for safety, belonging, and esteem were not well addressed in the beginning months of 
the pandemic, and many of these needs continue to be chronically unfulfilled. ${ }^{20,21}$ Major investments in infrastructure and cross-sector partnerships are vital so communities can immediately respond to future crises without compromising the health and well-being of essential workers, including primary care clinicians. ${ }^{4,5,19,22}$ As others have argued, the inadequacy of such investments previously should now be seen as a moral failure, as we have known about their importance for protecting the public's health for decades. ${ }^{23}$

There are limitations to this pragmatic qualitative analysis. Unfortunately, we were not able to specify practice and demographic details for those who actually participated in the sessions. Although we have information on those who registered for the ECHO program, it is possible some people participated without registering. Further, as many individuals used only their first names, initials, or device names as their Zoom screen names, it was impossible to accurately assign statements to individual participants. Large numbers of unique screen names participated in the chat box each week, however, and we believe the questions and comments they made are indicative of the needs of the participants.

Despite these limitations, our analysis uses a novel and timely data source to show that connection between peers is a major need for clinicians in times of crisis. The importance of sharing the experience of practicing in an uncertain and rapidly changing environment was evident in the many comments and questions participants made not only to the presenters, but also in public comments to each other. We found that participants created a virtual community that bolstered many of their core needs. Telementoring programs that provide opportunities for robust participant interactions - with their ability to both disseminate evidence-based information quickly and provide peer-to-peer support—should continue to play an integral role in responding to health crises in the future. Additionally, we recommend evaluators and researchers consider the potential for chat box data in identifying emerging needs of health care learners in future $\mathrm{ECHO}$ and other virtually based programs.

Read or post commentaries in response to this article.

Key words: COVID-19; primary health care; clinician well-being; project ECHO; professional practice; support groups; communication; health informatics; continuing medical education

Submitted April 13, 2021; submitted, revised, July 13, 2021; accepted August 17, 2021.

Acknowledgments: We would like to recognize additional individuals who were instrumental to the creation and implementation of the COVID-19 ECHO in Oregon, including Tuesday Graham, BS; Miriam Wolf, BS; Bruce Goldberg, MD; Glenn Rodriguez, MD; Dana Hargunani, MD; Thomas Jeanne, MD; and Jennifer Vines, MD. We also thank Melinda Davis, PhD; Jennifer Coury, MA; and Mary Patzel, MBA for their feedback and edits on the manuscript.

Funding support: The State of Oregon, through the Oregon Health Authority, funded the COVID-19 ECHO.

Disclaimer: The views expressed are solely those of the authors and do not necessarily represent official views of the authors' affiliated institutions or funder.

\section{Supplemental materials}

\section{References}

1. Kamerow D. Covid-19: Don't forget the impact on US family physicians. BMJ. 2020;368:m1260. 10.1136/bmj.m1260

2. Hallas L, Hatibie A, Majumdar $S$, et al. Variation in US states' responses to COVID-19. Blavatnik School of Government, University of Oxford. Published Dec 2020. Accessed Mar 1, 2021. https://www.bsg.ox.ac.uk/sites/default/ files/2020-12/BSG-W/P-2020-034-v2_0.pdf

3. Osterholm MT. Preparing for the next pandemic. N Engl J Med. 2005;352(18): 1839-1842. 10.1056/NEJMp058068

4. Ali MK, Shah DJ, Del Rio C. Preparing primary care for COVID-20. J Gen Intern Med. 2020;Jun 9;1-2. 10.1007/s11606-020-05945-5

5. Krist AH, DeVoe JE, Cheng A, Ehrlich T, Jones SM. Redesigning primary care to address the COVID-19 pandemic in the midst of the pandemic. Ann Fam Med. 2020;18(4):349-354. 10.1370/afm.2557

6. Hale AJ, Ricotta DN, Freed J, Smith CC, Huang GC. Adapting Maslow's hierarchy of needs as a framework for resident wellness. Teach Learn Med. 2019; 31(1):109-118. 10.1080/10401334.2018.1456928

7. Hale AJ, Ricotta DN, Freed JA, Smith CC, Huang GC. Comparing 2 adapted Maslow's hierarchy of needs frameworks on physician wellness. Am J Med. 2020;133(9):e532-e533. 10.1016/j.amjmed.2020.02.030

8. Shapiro DE, Duquette C, Abbott LM, Babineau T, Pearl A, Haidet P. Beyond burnout: a physician wellness hierarchy designed to prioritize interventions at the systems level. Am J Med. 2019;132(5):556-563.

9. Maslow A. A Theory of human motivation. Psychol Rev. 1943;50(4):370-396.

10. Arora S, Kalishman S, Dion D, et al. Partnering urban academic medical centers and rural primary care clinicians to provide complex chronic disease care. Health Aff (Millwood). 2011;30(6):1176-1184. 10.1377/hlthaff.2011.0278

11. Arora S, Kalishman S, Thornton K, et al. Project ECHO (Project Extension for Community Healthcare Outcomes): a national and global model for continuing professional development. J Contin Educ Health Prof. 2016;36(Suppl 1): S48-S49. 10.1097/CEH.0000000000000097

12. McDonnell MM, Elder NC, Stock R, Wolf M, Steeves-Reece A, Graham T. Project ECHO integrated within the Oregon Rural Practice-Based Research Network (ORPRN). J Am Board Fam Med. 2020;33(5):789-795.

13. Steeves-Reece AL, Elder NC, Graham TA, et al. Rapid deployment of a statewide COVID-19 ECHO program for frontline clinicians: early results and lessons learned. J Rural Health. 2021;37(1):227-230. 10.1111/jrh.12462

14. Robinson S, Angier $\mathrm{H}$, Pierce $\mathrm{K}$, et al. Responding to community questions during a pandemic: development of a COVID-19 inquiry group. In: COVID-19 Collection: Primary Care Pandemic Response, Annals of Family Medicine. Published Mar 28, 2020. Accessed Mar 1, 2021. https://www.annfammed.org/ content/covid-19-collection-primary-care-pandemic-response

15. Crabtree B, Miller W, eds. Doing Qualitative Research. 2nd ed. Sage; 1999.

16. Green LA, Fryer GE Jr, Yawn BP, Lanier D, Dovey SM. The ecology of medical care revisited. N Engl J Med. 2001;344(26):2021-2025.

17. Mexico PEUON. Project ECHO COVID-19 response. Partner portal. University of New Mexico School of Medicine. Published 2020. Accessed Mar 1, 2021. https://hsc.unm.edu/echo/institute-programs/covid-19-response/

18. Project ECHO: get involved. University of New Mexico School of Medicine. Published 2020. Accessed Mar 1, 2021. https://hsc.unm.edu/echo/get-involved/

19. Geyman JP. Beyond the COVID-19 pandemic: the urgent need to expand primary care and family medicine. Fam Med. 2021;53(1):48-53.

20. Fish JN, Mittal M. Mental health providers during COVID-19 : essential to the US public health workforce and in need of support. Public Health Rep. 2021;136(1):14-17. 10.1177/0033354920965266

21. Glenza J. PPE shortage could last years without strategic plan, experts warn. Kaiser Health News. Published Aug 17, 2020. Accessed Mar 1, 2021. https://khn. org/news/ppe-shortage-could-last-years-without-strategic-plan-experts-warn/

22. Mason DJ, Friese CR. Protecting health care workers against COVID-19and being prepared for future pandemics. JAMA Health Forum. 2020;1(3): e200353.

23. Smith MJ, Upshur REG. Ebola and learning lessons from moral failures: who cares about ethics? Public Health Ethics. 2015;8(3):305-318. 